\title{
3D Printing Mechanically Robust and Transparent Polyurethane Elastomers for Stretchable Electronic Sensors
}

Shuqiang Peng, ${ }^{\dagger, t}$ Yuewei Li, ${ }^{\dagger, t}$ Lixin Wu, ${ }^{* \dagger}$ Jie Zhong, ${ }^{* \dagger}$ Zixiang Weng, ${ }^{\dagger}$ Longhui Zheng, ${ }^{\dagger}$ Zhi Yang, ${ }^{\dagger}$ and Jia-Tao Miao ${ }^{*}{ }^{\dagger}$

$\uparrow$ CAS Key Laboratory of Design and Assembly of Functional Nanostructures, Fujian Key Laboratory of Nanomaterials, Fujian Institute of Research on the Structure of Matter, Chinese Academy of Sciences, Fuzhou 350002, People's Republic of China

¥University of Chinese Academy of Sciences, Beijing 100049, People’s Republic of China

Corresponding Authors

*E-mail: 1xwu@fjirsm.ac.cn (L.W.)

*E-mail: zhongjie@fjirsm.ac.cn (J.Z.)

*E-mail: jiataomiao@outlook.com (J.-T.M.) 


\section{Index}

Scheme S1. Illustration of the synthetic strategy for PUAs................................................ S-3

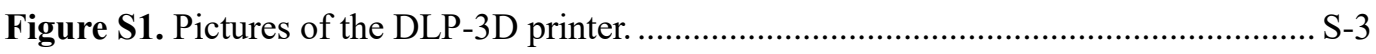

Figure S2. (a) FTIR spectra of the NCO terminated prepolymers and PUAs synthesized with different polyols. (b) FTIR spectra for $\mathrm{C}=\mathrm{O}$ stretching band $\left(1800-1680 \mathrm{~cm}^{-1}\right)$ of synthesized PUAs.

Figure S3. ${ }^{1} \mathrm{H}$ NMR spectra of PPTMGA, PPPGA and PPCLA in DMSO- $d_{6}$ S-5

Figure S4. (a) Viscosity of PUAs versus shear rates. (b) Viscosity of PUAs-40 versus shear rates at $25^{\circ} \mathrm{C}$ S-6

Figure S5. 3D-printed finger guards (PPTMGA-40 without photoabsorber) with rough surfaces. S-6

Figure S6. UV-vis absorption spectrum of BBOT in acetonitrile. S-6

Figure S7. (a) 3D-printed cylinders (PPTMGA-40) of different diameters $(1.2 \mathrm{~mm}, 0.8 \mathrm{~mm}$, $0.4 \mathrm{~mm}$ and $0.2 \mathrm{~mm}$ ). (b) Optical images for printed cylinders of different diameters. S-7

Figure S8. The picture of Taber abrasion machine................................................ S-8

Figure S9. The weight loss of PUAs-40 with Taber abrasion. ................................ S-8

Figure S10. Retention of maximum stress (PPTMGA-40) during 100 compression cycles of $80 \%$ strain. S-9

Figure S11. The tensile stress-strain curve of ionic hydrogel.................................. S-9

Figure S12. UV-vis absorption spectra of hydrogel film and hydrogel/PPTMGA-40 hybrid.

Figure S13. (a) FTIR spectra of PPTMGA-40 resin before and after post curing. (b) Schematic illustration of covalent bonds between hydrogel and PPTMGA-40. S-10

Figure S14. Electrical resistance recoverability of hydrogel-coated PPTMGA-40 sensor in response to compression and release

Figure S15. Resistance change of hydrogel-coated PPTMGA-40 sensor versus compressive stress. The pressure sensitivity $(S)$ is calculated as the slope of the curves. S-11

Table S1. Cure depth of PPTMGA-40, PPPGA-40 and PPCLA-40 with different concentrations of photoabsorber BBOT. 

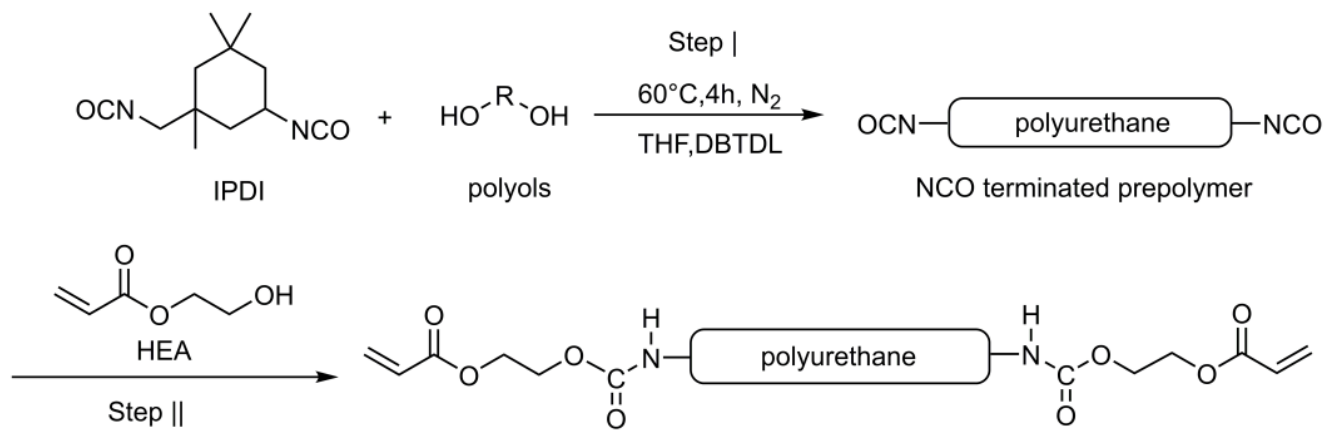

polyurethane acrylate oligomers

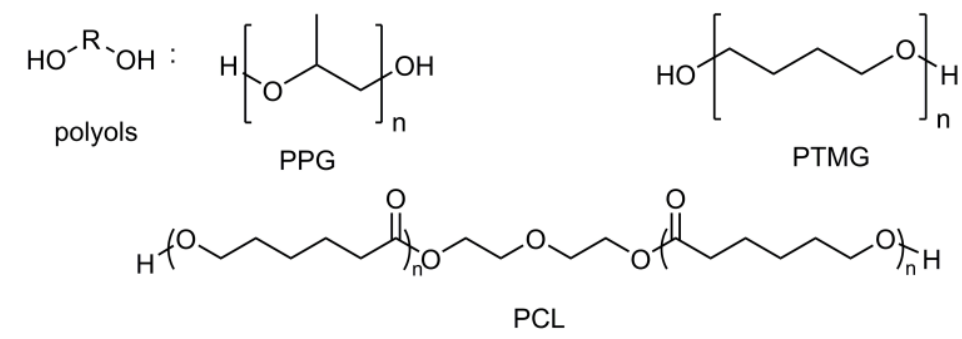

Scheme S1. Illustration of the synthetic strategy for PUAs.

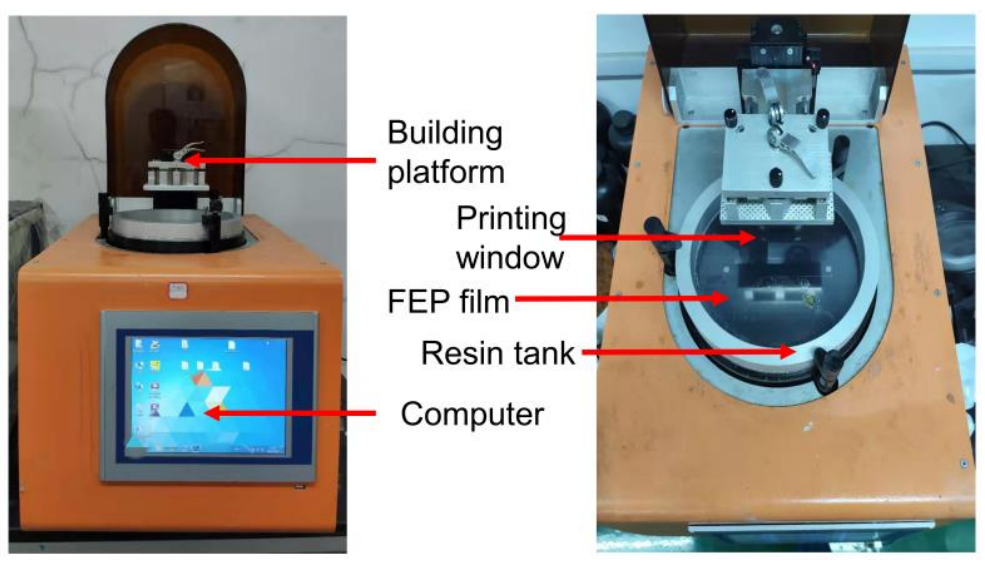

Figure S1. Pictures of the DLP-3D printer. 

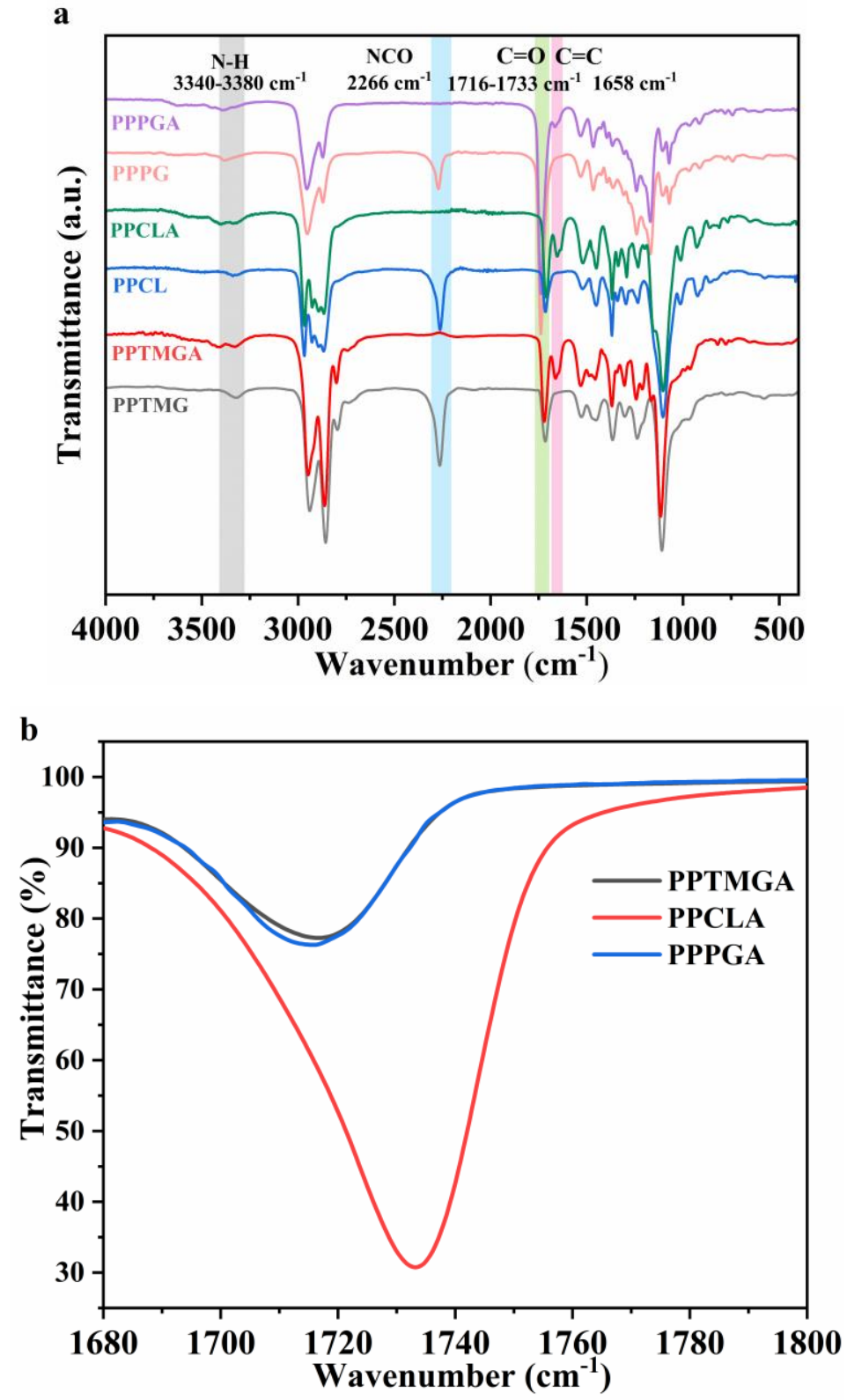

Figure S2. (a) FTIR spectra of the NCO terminated prepolymers and PUAs synthesized with different polyols. (b) FTIR spectra for $\mathrm{C}=\mathrm{O}$ stretching band $\left(1800-1680 \mathrm{~cm}^{-1}\right)$ of synthesized PUAs. 


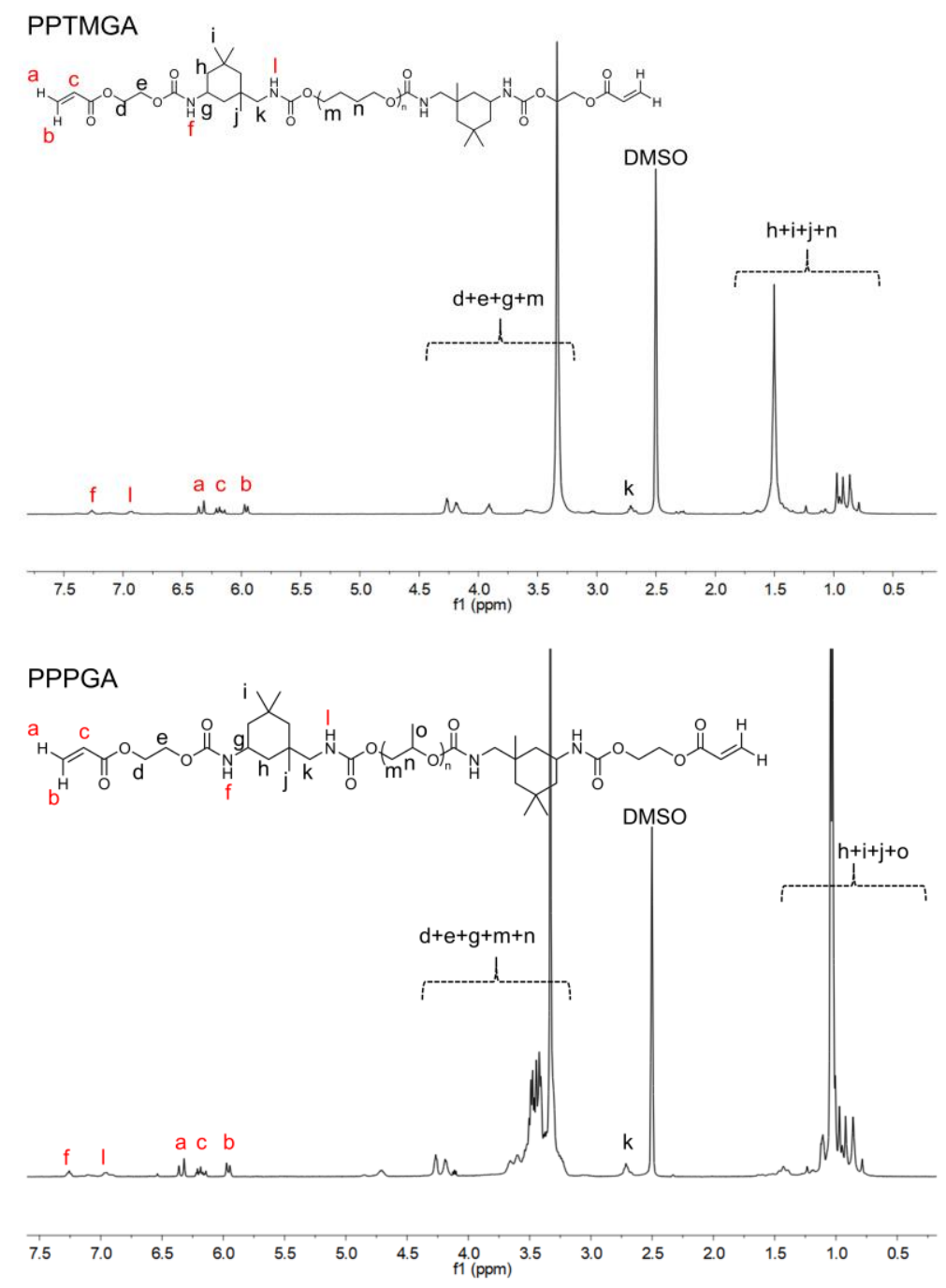

PPCLA

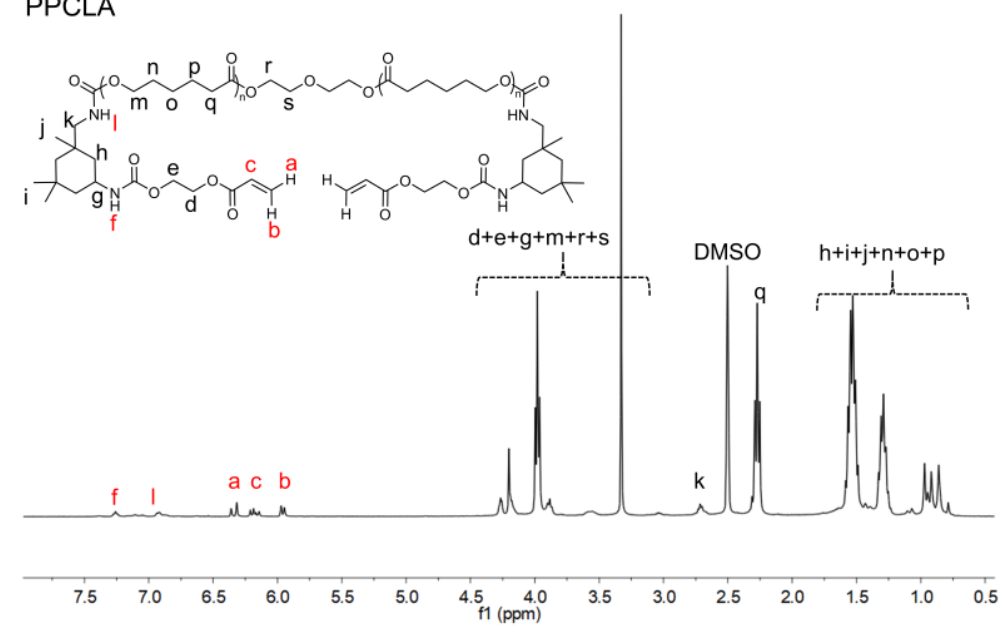

Figure S3. ${ }^{1} \mathrm{H}$ NMR spectra of PPTMGA, PPPGA and PPCLA in DMSO- $d_{6}$. 

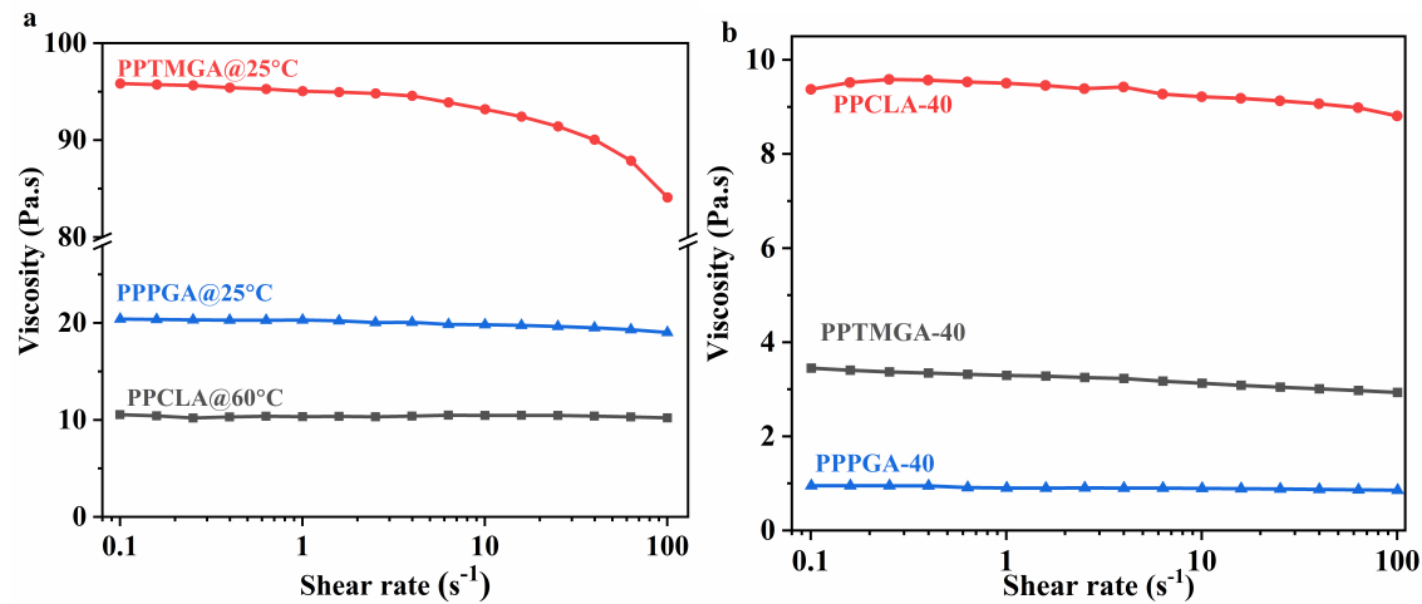

Figure S4. (a) Viscosity of PUAs versus shear rates. (b) Viscosity of PUAs-40 versus shear rates at $25^{\circ} \mathrm{C}$.

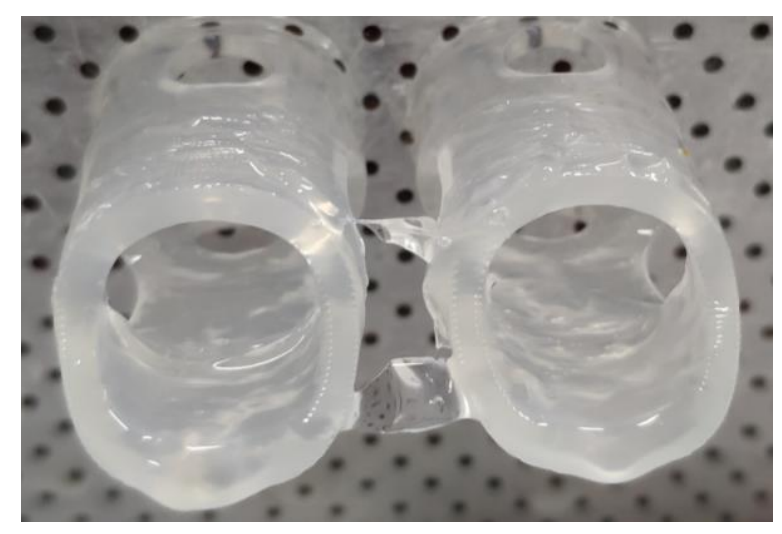

Figure S5. 3D-printed finger guards (PPTMGA-40 without photoabsorber) with rough surfaces.

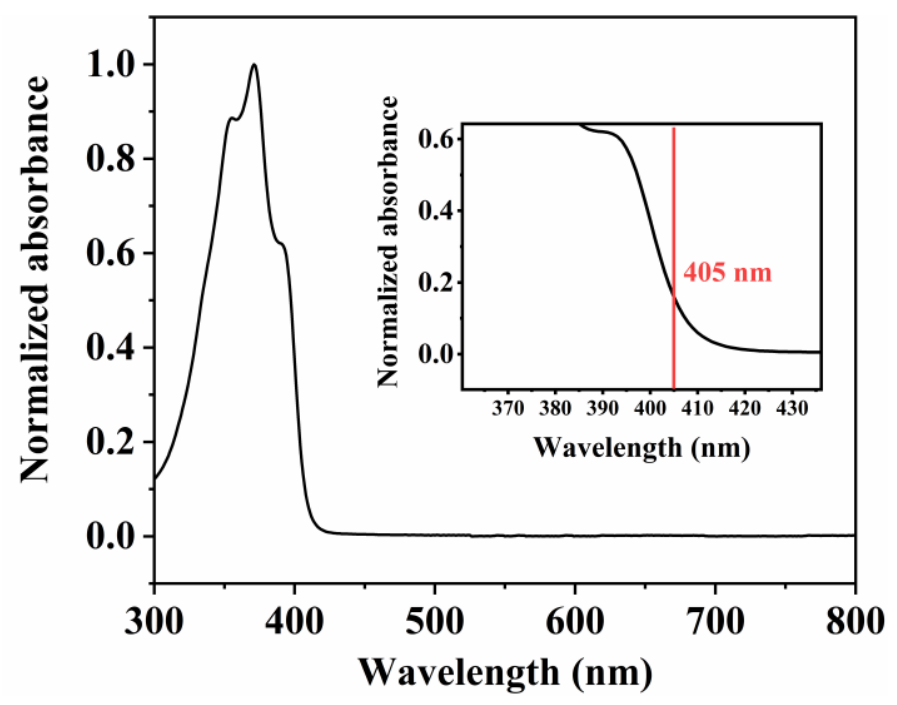

Figure S6. UV-vis absorption spectrum of BBOT in acetonitrile. 


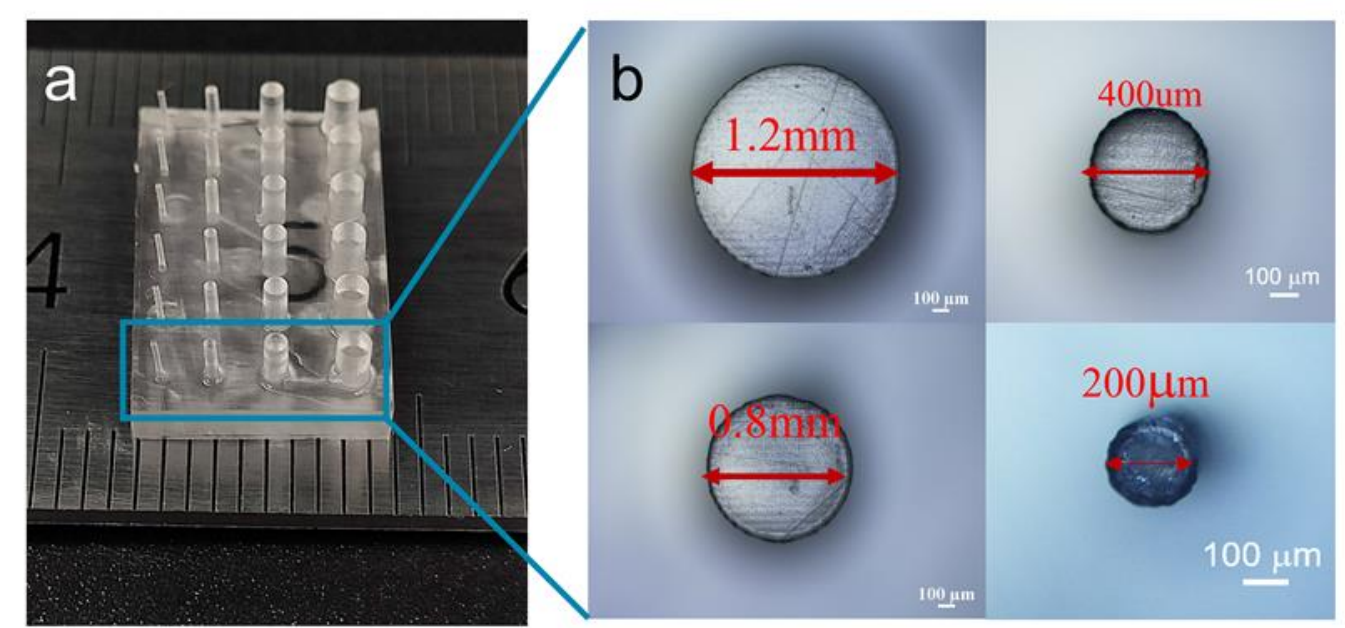

Figure S7. (a) 3D-printed cylinders (PPTMGA-40) of different diameters $(1.2 \mathrm{~mm}, 0.8 \mathrm{~mm}, 0.4 \mathrm{~mm}$ and $0.2 \mathrm{~mm})$. (b) Optical images for printed cylinders of different diameters. 


\section{Abrasion resistance test}

The abrasion tests of polyurethane elastomers were conducted on Taber abrasion machine (Figure S8) according to ASTM D-4060. The mechanical durability performance is evaluated by the weight loss $(\mathrm{mg})$ of the material under periodic testing. The abrasion machine is equipped with two $250 \mathrm{~g}$ abrading arms and additionally carries two $500 \mathrm{~g}$ weights at a rotation speed of $60 \pm 2 \mathrm{r} / \mathrm{min}$. The samples were circular shape with diameter of $100 \mathrm{~mm}$, thickness of $3 \mathrm{~mm}$ and a $6 \mathrm{~mm}$ hole was punched in the middle to fix it on the rotational platform of the Taber machine. The weight loss was measured after 200 and 500 cycles and plotted in Figure S9.

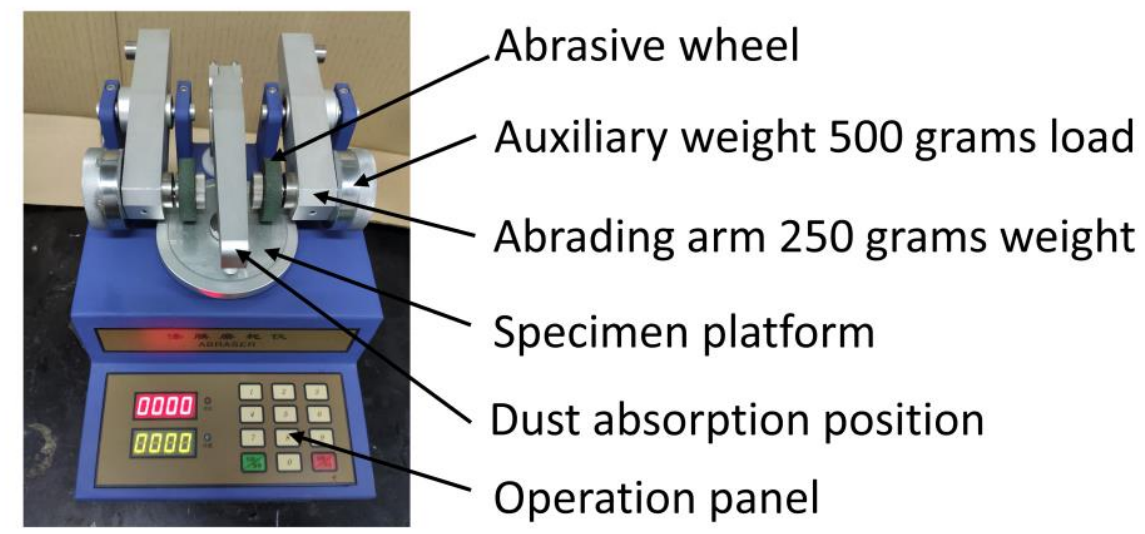

Figure S8. The picture of Taber abrasion machine.

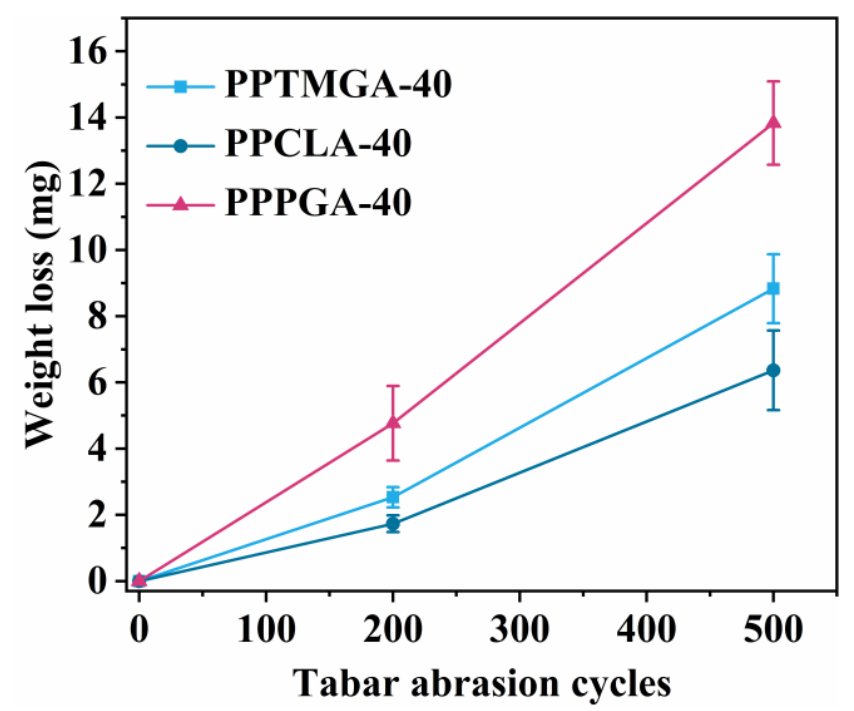

Figure S9. The weight loss of PUAs-40 with Taber abrasion. 


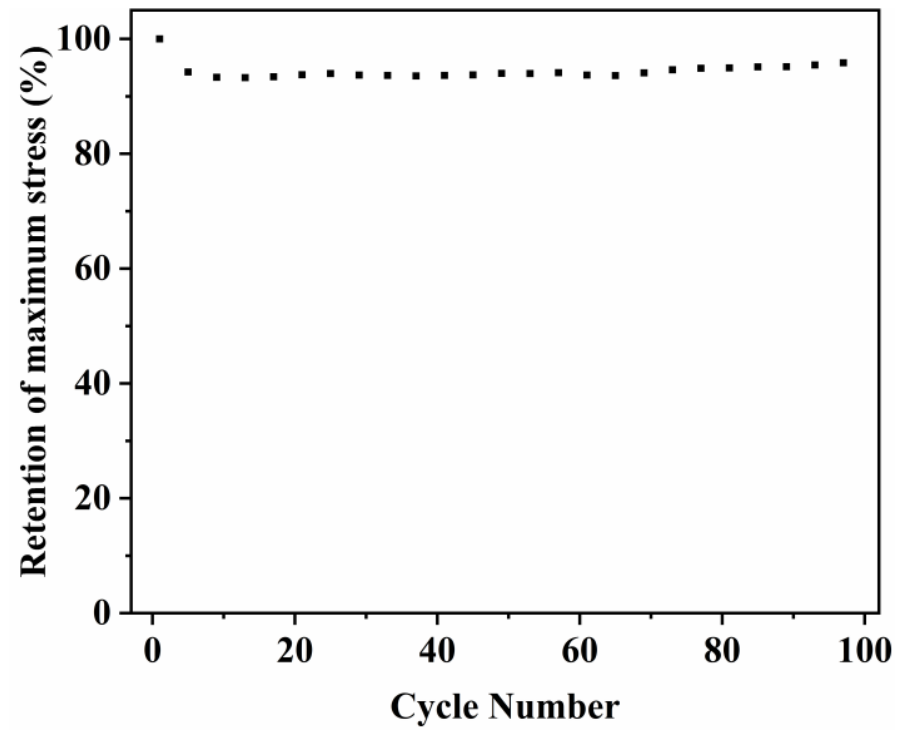

Figure S10. Retention of maximum stress (PPTMGA-40) during 100 compression cycles of $80 \%$ strain.

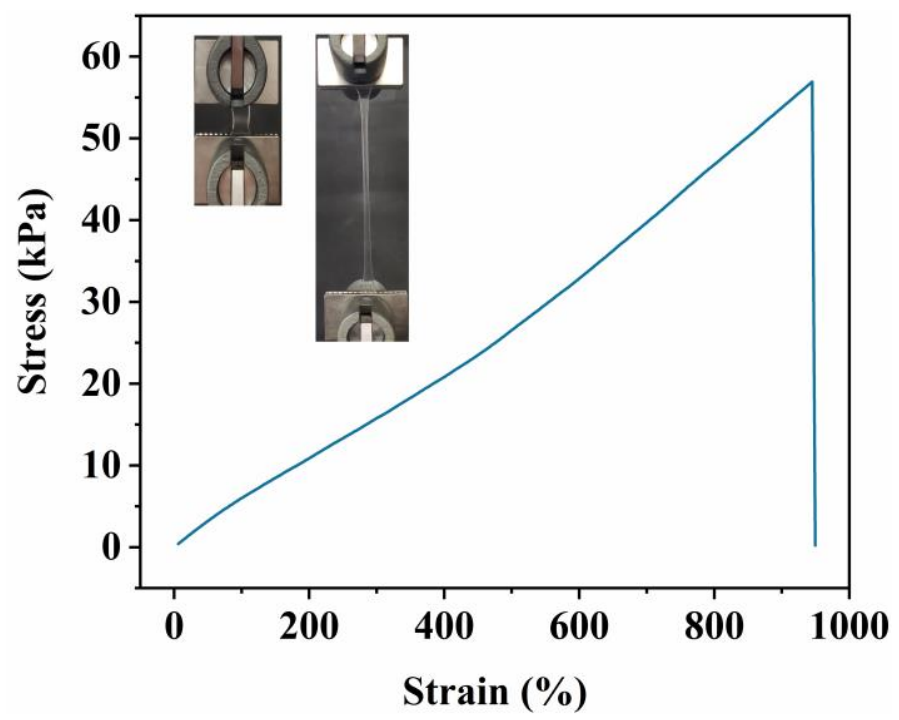

Figure S11. The tensile stress-strain curve of ionic hydrogel. 


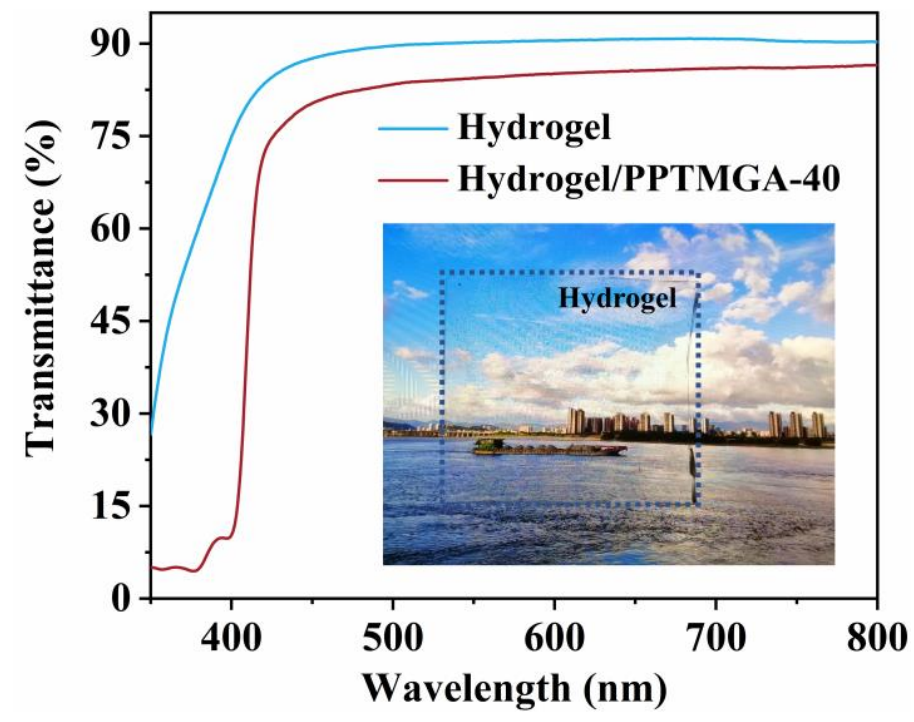

Figure S12. UV-vis absorption spectra of hydrogel film and hydrogel/PPTMGA-40 hybrid.

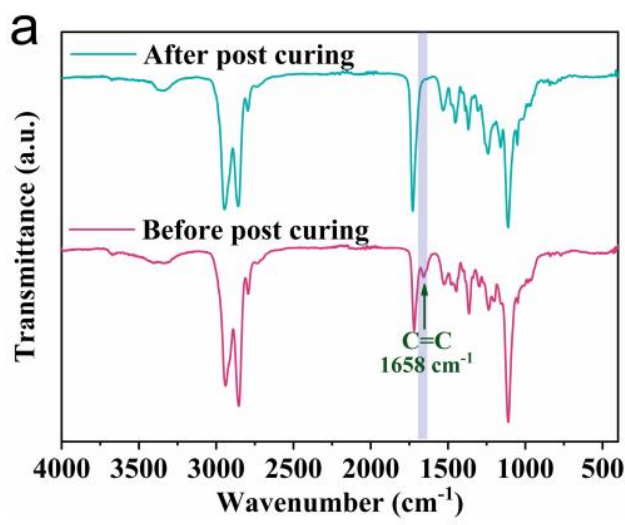

b

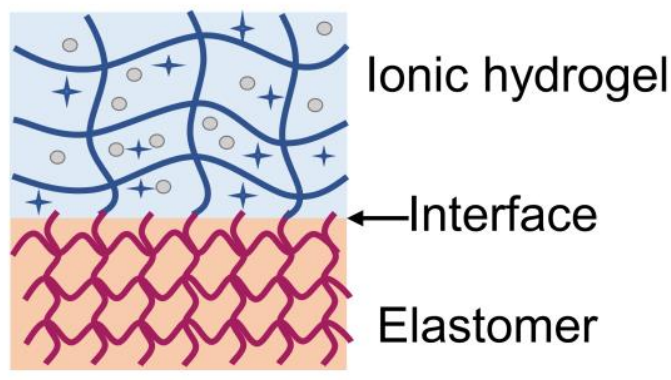

Figure S13. (a) FTIR spectra of PPTMGA-40 resin before and after post curing. (b) Schematic illustration of covalent bonds between hydrogel and PPTMGA-40. 


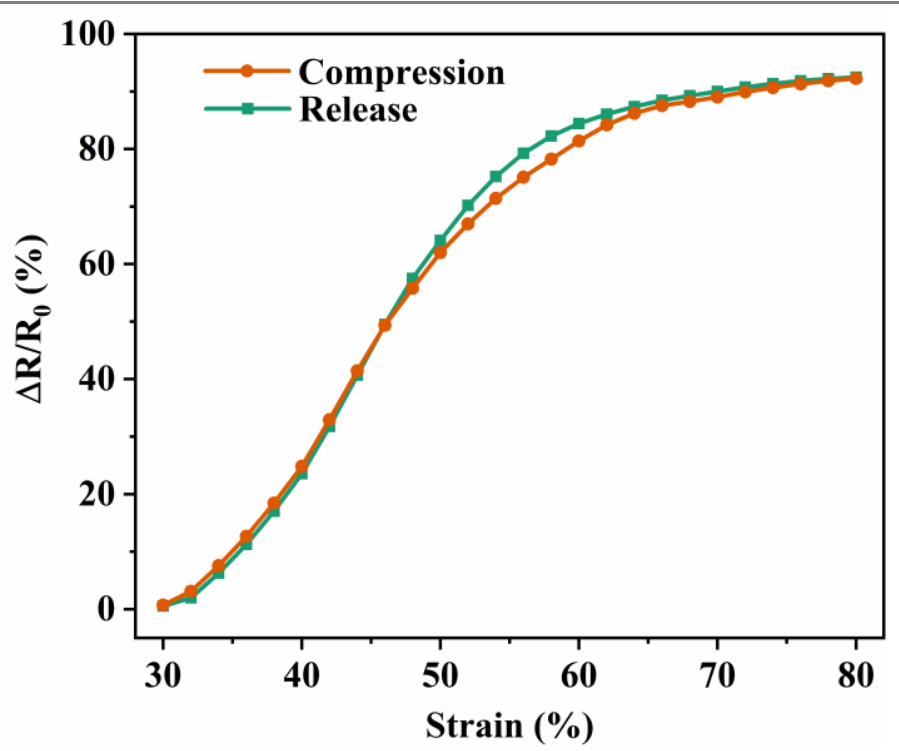

Figure S14. Electrical resistance recoverability of hydrogel-coated PPTMGA-40 sensor in response to compression and release.

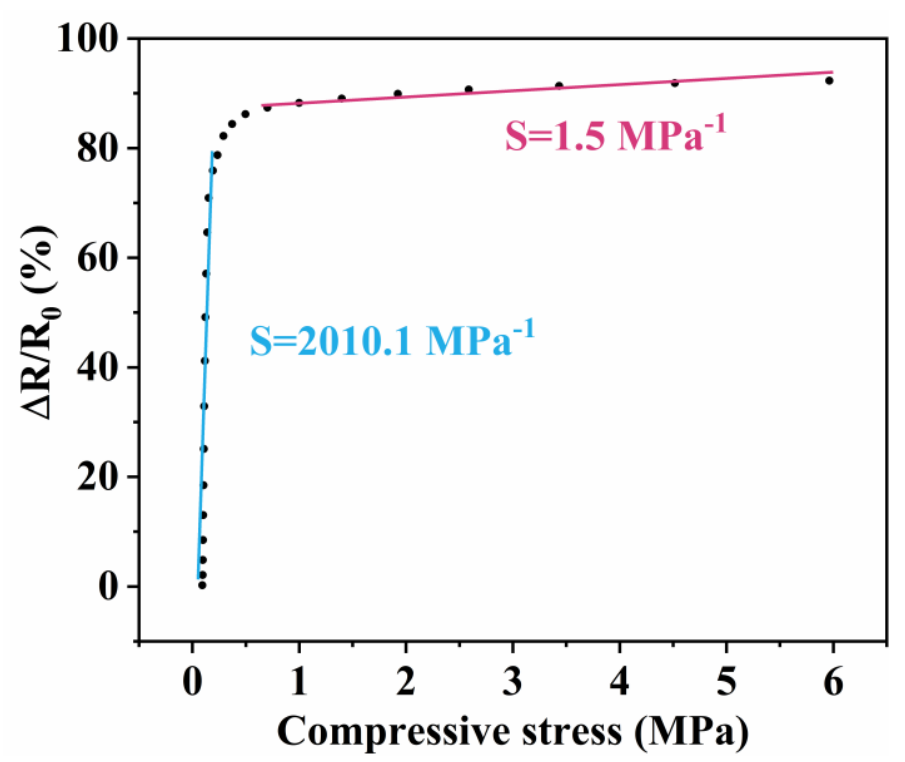

Figure S15. Resistance change of hydrogel-coated PPTMGA-40 sensor versus compressive stress. The pressure sensitivity $(S)$ is calculated as the slope of the curves. 
Table S1. Cure depth of PPTMGA-40, PPPGA-40 and PPCLA-40 with different concentrations of photoabsorber BBOT.

\begin{tabular}{cccc}
\hline \multirow{2}{*}{ BBOT $(\mathrm{wt} \%)$} & \multicolumn{3}{c}{$h_{\mathrm{p}}(\mathrm{mm})$} \\
\cline { 2 - 4 } & PPTMGA-40 & PPPGA-40 & PPCLA-40 \\
\hline 0 & 1.014 & 1.136 & 0.930 \\
0.05 & 0.325 & 0.376 & 0.290 \\
0.1 & 0.223 & 0.256 & 0.187 \\
\hline
\end{tabular}

